FORMATION Formation emploi

Revue française de sciences sociales

138 | Avril-Juin 2017

La professionnalisation dans l'enseignement supérieur : formes et effets variés

\title{
La professionnalisation des universités par la formation continue des adultes : une comparaison Québec-France
}

The professionalization of the universities through their lifelong learning for adults : a comparison Quebec-France

Die Professionalisierung der Universitäten durch Erwachsenenfortbildung : ein Vergleich zwischen Quebec und Frankreich

La profesionalización de las universidades por la formación continua de los adultos : una comparación Québec-Francia

Pierre Doray et Christelle Manifet

\section{(2) OpenEdition Journals}

Édition électronique

URL : http://journals.openedition.org/formationemploi/5101

DOI : 10.4000/formationemploi.5101

ISSN : 2107-0946

Éditeur

La Documentation française

Édition imprimée

Date de publication : 17 juillet 2017

Pagination : 139-163

ISSN : 0759-6340

Référence électronique

Pierre Doray et Christelle Manifet, «La professionnalisation des universités par la formation continue des adultes : une comparaison Québec-France », Formation emploi [En ligne], 138 | Avril-Juin 2017, mis en ligne le 10 juillet 2019, consulté le 30 octobre 2020. URL : http://journals.openedition.org/ formationemploi/5101; DOI : https://doi.org/10.4000/formationemploi.5101 


\title{
La professionnalisation des universités par la formation continue des adultes : une comparaison Québec-France
}

\author{
Pierre Doray \\ Professeur de sociologie, CIRST (Centre interuniversitaire de recherche sur la science et la \\ technologie), université de Québec à Montréal (UQAM) \\ Christelle Manifet \\ Maître de conférences en sociologie, CERTOP (Centre d'Étude et de Recherche Travail, \\ Organisation, Pouvoir, UMR CNRS 5044), université Toulouse 2 Jean Jaurès (UT2J)
}

Résumé

La professionnalisation des universités par la formation continue des adultes : une comparaison Québec-France

Cet article interroge la professionnalisation des universités sur la base de leurs activités d'enseignement pour adultes. Il le fait de manière comparative, entre le Québec et la France, deux situations apparemment contrastées. Suivant l'analyse sociétale et les méthodes de la sociologie des catégories de l'intervention publique, l'analyse dégage deux trajectoires singulières de développement d'une offre universitaire à destination des adultes. En plus de révéler les catégories sociétales de la formation continue, cette méthode permet d'être attentif à ce qui les lie autant qu'à ce qui les éloigne. L'article montre que la professionnalisation des universités engage un système de conventions et de distribution des cartes entre Université, État, acteurs du travail et de l'emploi et individus en retour aux études et/ou au travail.

Mots-clés : enseignement supérieur, accès à l'enseignement supérieur, politique de la formation professionnelle, université, professionnalisation de l'enseignement, formation des adultes, France, Québec, comparaison internationale

Abstract

The professionalization of the universities through their lifelong learning for adults: a comparison Quebec-France

This article examines the professionalization process of universities through their adult education practices. It does so in a comparative way, between Quebec and France, two apparently contrasted situations. Following the societal analysis and the methods of the sociology of public policy categories, the analysis distinguishes two specific development paths for the university education supply addressing adult demand. While revealing the 
societal categories of lifelong learning, this method allows us to be attentive to what connects them as much as what depart from them. The article demonstrates that the professionalization of the universities engages a system of conventions, specific in each society by a local and historical configuration of action, between the University, the State, Socioeconomics partners and Individuals going back to study and/or at work.

Keywords: higher education, access to higher education, CVT policy, university, professionalisation of teaching, adult training, France, Quebec, international comparison Journal of Economic Literature: I 23

Traduction : Auteur-e-s.

La professionnalisation des universités est explorée sous différentes voies. Une première voie est celle de l'introduction de profils, pratiques et discours managériaux dans la gestion des établissements (Kolsaker, 2014). Une seconde voie examine l'orientation croissante des étudiants vers les formations professionnelles (Crespo, 2013 ; Landrier et Nakhili, 2012). Une troisième, à l'intérieur des universités, examine la construction d'une offre de formation associée à des emplois (Doray, Tremblay et Groleau, 2015 ; Wittorski, 2012). Est aussi examinée l'incorporation, dans les programmes universitaires, d'activité de préparation à l'emploi comme les stages (Beaupère, Bosse, et Lemistre 2014), afin de favoriser l'insertion professionnelle des personnes diplômées.

Il existe une autre voie de professionnalisation, la formation des adultes, identifiée sous différentes appellations : formation continue, éducation permanente, perfectionnement professionnel, etc. ${ }^{1}$ L'articulation entre formation, travail et emploi semble ici une évidence du fait que les expériences sont synchrones (formation et emploi) ou prennent la forme d'un retour aux études (emploi vers formation). La présence d'adultes en formation à l'université, le plus souvent en lien à l'emploi, n'est-elle pas un signe immédiat du caractère professionnel des universités ? Il convient d'aller plus loin en s'intéressant, d'une part, à la manière dont les universités répondent aux besoins du marché de l'emploi ainsi qu'aux besoins d'un public adulte et d'autre part, en regardant d'un peu plus près les types de contenu de formation ainsi que les aménagements réalisés pour accueillir ces adultes.

Les adultes en formation rassemblent quelques caractéristiques qui spécifient leur rapport aux études, en particulier, leur âge. Ils sont entrés dans des cycles de vie où les différentes sphères se déploient (entrée dans la vie active, installation en couple, naissance des enfants, etc.). Des aménagements du " contenu » des formations comme des "parcours " d'études (Lemistre, 2016) s'imposent pour tenir compte de la conciliation avec d'autres activités

1. L' appellation « adulte " relève d'un choix scientifique. Elle n'est, la plupart du temps, pas utilisée par les acteurs. Les catégories " gestionnaires» (Demazière, 2002) identifient les adultes sous d'autres dénominations, par public (étudiant, stagiaire, auditeur...) et/ou par type de formation (formation continue, éducation permanente...). 
de la vie d'adulte (assurer sa subsistance, avoir des responsabilités professionnelles et familiales). Les aménagements de contenu s'étendent de la formation sur mesure non créditée et construite conventionnellement avec des employeurs ou des ordres professionnels jusqu'à l'offre diplômante classique ouverte à la formation continue, en passant par les dispositifs de validation de l'expérience pour l'accès ou le retour aux études supérieures ou pour l'obtention de diplômes. Les aménagements de parcours concernent les calendriers et les parcours d'études avec l'organisation en trimestres, les études à temps partiel et les cours du soir ou les congés pour formation et les formations courtes.

Pour réfléchir à cette voie singulière de professionnalisation et à ses formes concrètes, on s'appuie sur la comparaison internationale. Les diagnostics exploitant la comparaison comme outil de décision, établis par l'Union européenne, l'UNESCO (Organisation des nations unies pour l'éducation, la science et la culture) ou l'OCDE (Organisation de coopération de développement économiques), décrivent des engagements variables des systèmes éducatifs et des universités en matière de formation pour adultes. Ainsi, en France, cet engagement serait plus restreint tant du point de vue des compétences et de l'accès des adultes à la formation que du rôle joué par le système scolaire et universitaire, en comparaison avec d'autres pays, comme le Canada et en son sein la province de Québec, qui connaissent une participation des adultes nettement plus élevée à l'enseignement universitaire $^{2}$. La recherche part de cette représentation impressionniste de départ : la présence des adultes aux études universitaires serait nettement plus faible en France qu'au Québec ${ }^{3}$. La comparaison pourrait consister à mesurer des degrés différenciés de professionnalisation à partir d'un petit nombre d'indicateurs. Elle négligerait cependant des effets de systèmes et créerait une illusion d'égalité. Il convient donc d'introduire une réflexion sur la comparabilité des données (Von Rosenbladt, 2010), ce à quoi l'analyse comparée qui suit tente de s'atteler.

Cette comparaison s'inspire du principe de l'approche sociétale (Maurice, 1989) selon lequel la constitution d'un objet social se réalise dans l'interdépendance entre les composantes structurelles d'une société et le travail social des acteurs. Cette interdépendance génère des cohérences ou des configurations spécifiques (Maurice, Sellier et Silvestre, 1982) et donne corps à des catégorisations particulières (Dubar et al., 2003). Récemment, Éric Verdier a proposé le concept de régime national d'éducation et de formation pour embrasser des « configurations d'acteurs (...) articulées à des organisations et des institutions "

2. Par exemple, en matière de compétences acquises par les adultes (15-65 ans) tant en littératie qu'en numératie, la France obtient des scores inférieurs à la moyenne des pays de l'OCDE, tandis que le Canada se situe plutôt dans la moyenne (OCDE, 2013).

3. La réalisation de l'étude n'aurait pas été possible sans le soutien de différents organismes : le Conseil de recherche en sciences humaines du Canada (CCRSHC), le Fonds Recherches Québec-société et culture (FRQCS), le Conseil international d'études canadiennes et le Gouvernement du Canada, le Laboratoire d'excellence Structuration des mondes sociaux (Labex SMS) de Toulouse et le gouvernement français. Nous désirons également remercier la rédaction de Formation Emploi ainsi que les différents évaluateurs sollicités pour leurs précieuses remarques. 
(Verdier, 2008, p. 203), ainsi que des conventions de formation (méritocratique, professionnelle ou marchande). Pour saisir ces configurations et ces catégorisations nationales, ont été exploitées les méthodes de la sociologie des catégories de l'intervention publique (Dubois, 2003 et 2012) : la réalité des usages sociaux de l'université n'est pas indépendante des catégorisations que des espaces sociaux divers, à l'intérieur de sociétés particulières et notamment les espaces politiques institués, entretiennent à leur égard. L'histoire croisée a aussi permis de ne pas sur-interpréter les dissemblances entre les cas et d'être attentif aux similitudes (Gally, 2012).

Le matériau relève de différentes sources. La première est composée des sources indirectes issues de la littérature scientifique spécialisée sur la formation des adultes à l'université, dont des enquêtes réalisées dans les années 1970 et 1980, afin de dégager une profondeur historique. Diverses archives institutionnelles ont ensuite été dépouillées pour éclairer les contextes politiques et sociaux d'énonciation et de réception de décisions ayant trait à la formation des adultes à l'université, tant à l'échelle des ministères et des gouvernements (lois, discours programmatiques, témoignages écrits d'élites politiques et administratives...) qu'à l'échelle des établissements (comptes-rendus et procès-verbaux d'instances décisionnelles de deux universités ayant fait l'objet d'études de cas approfondies). Des études de cas sur la situation de l'éducation des adultes ont enfin été menées dans différentes universités françaises et québécoises. Elles ont permis de mieux comprendre les modalités d'insertion des adultes dans les universités, l'ancrage réglementaire et organisationnel de l'activité de formation pour adultes et les pratiques des professionnels de l'université. Par ailleurs, les données administratives sur les inscriptions des étudiants ont été exploitées.

L'argument suit deux objectifs. Le premier pointe les éléments structurants de la formation universitaire des adultes dans les sociétés québécoise, puis française, à l'appui de deux questions simples, l'une sur les structures cognitives, normatives et institutionnelles de l'engagement des universités dans cette activité, l'autre sur les caractéristiques du service rendu aux adultes dans les établissements (I et II). Le second objectif est de tirer de ces configurations sectorielles et sociétales des éléments d'interprétation plus généraux sur les voies de professionnalisation de l'université et ses conditions (III). 


\section{Encadré 1 : Les études de cas réalisées}

2007 : La formation continue dans les universités françaises, cinq établissements d'enseignement supérieur (sites web, documentation institutionnelle, entretiens).

2011 : La formation tout au long de la vie, enjeu de société, réponses universitaires et recomposition du service public d'enseignement supérieur : trois universités du Québec organisant de manière variée leur activité de formation continue (sites web, documentation institutionnelle, entretiens, observations).

2015-2016 : L'adéquation entre la formation et l'emploi : le cas de la formation sur-mesure dans l'enseignement supérieur du Québec (collèges d'enseignement général et professionnel CEGEP - et universités).

2015-2017 : L'université et ses publics. Les adultes. Monographie d'une université régionale en France (traitement des archives de l'université, observations de chaînes d'inscription, entretiens auprès des services, entretiens individuels et collectifs avec des inscrits adultes).

\section{Au Québec, une professionnalisation endogène}

Au Québec, la professionnalisation de l'université répond à une dynamique qui sera qualifiée d'endogène car la formation des adultes y est considérée comme un segment de la politique éducative et de l'activité des universités (1.1.) ; l'articulation au monde $\mathrm{du}$ travail et de l'emploi prend d'abord effet par l'intégration des publics adultes aux programmes crédités (1.2.) et se poursuit, depuis les années 1990, par l'essor des formations non créditées (1.3.).

\subsection{L'ambition de l'éducation permanente}

En 1960, est lancée la Révolution tranquille, période de profonde modernisation de la société québécoise et de l'action gouvernementale, avec la mise en place d'un Étatprovidence ${ }^{4}$. Le secteur public de l'éducation acquiert de la consistance avec la création du ministère de l'Éducation et du Conseil supérieur de l'Éducation et avec le développement du réseau des universités, passant de 8 à 18 établissements. Deux autres organismes consultatifs verront ensuite le jour, le Conseil des collèges et le Conseil des universités.

La démocratisation de l'accès à l'enseignement est le maître-mot de la réforme. Trois textes d'orientation de l'action éducative sont publiés rapidement : sur la formation pro-

4. Le gouvernement pose trois orientations fondamentales. Il se veut davantage interventionniste avec, par exemple, la nationalisation des entreprises d'électricité. Il se veut davantage pilote avec la création de divers organismes de planification, et davantage protecteur avec l'instauration de mesures de protection contre les aléas de la vie collective (régie des rentes du Québec, assurance maladie, etc.). 
fessionnelle et technique (Tremblay, 1962), sur l'éducation des adultes (Ryan, 1964), sur l'éducation dans la province de Québec (Parent, 1964). Il s'agit de démocratiser l'éducation et d'en faire un outil de mobilité sociale afin de permettre le rattrapage des francophones, mais aussi des milieux populaires, des femmes, des adultes. Augmenter la part de la population qui poursuivra des études supérieures est perçue comme la condition du développement social et économique de la société québécoise. Est aussi créé un système de prêts et de bourses et est envisagée la gratuité scolaire afin de faciliter l'accès aux universités 5 .

L'éducation des adultes est au cœur de ce projet éducatif. Le rapport Parent cherche à fédérer l'ensemble des segments de la formation des adultes, de l'éducation populaire aux cours crédités dans les établissements d'enseignement, en s'appuyant sur le concept de l'éducation permanente. Cette idée est très présente au Québec, alors que la 2e conférence internationale de l'UNESCO sur l'éducation des adultes s'est tenue à Montréal en 1960 et que l'Institut canadien de l'éducation des adultes en fait son cheval de bataille. L'éducation permanente devient le principe structurant du rapport que les individus doivent établir avec le monde de l'éducation. Dans cette perspective, l'université a une mission spécifique, car "dans une économie et une technologie en rapide transformation, l'université doit assumer une part de responsabilité dans l'éducation des adultes devenue toujours plus nécessaire. Il ne faut cependant pas lui demander de donner aux adultes un enseignement qu'elle ne donne pas à ses étudiants réguliers : cours secondaire ou pré-universitaire. Mais elle doit pouvoir étendre aux adultes, en cours du soir ou en cours d'été, son enseignement spécialisé» (Parent, 1964, p. 233). Ces deux orientations vont être au cœur de la mission de l'Université du Québec (UQ), créée en 1968, qui comportera à terme dix constituantes autonomes pour " assurer une réelle démocratisation de l'accès à l'enseignement supérieur et une coïncidence plus grande entre monde universitaire et société» (Ferretti, 1994, p. 39). La mission d'éducation permanente côtoie celles de l'enseignement et de la recherche.

\subsection{Dans les universités, un principe d'intégration des publics adultes}

Cette ligne directrice va se diffuser dans les universités du Québec pour deux raisons principales. D’une part, les universités plus anciennes ont transformé leur service d'extension de l'enseignement en service d'éducation des adultes. D'autre part, le réseau UQ va aussi s'engager dans la formation des adultes au nom de l'accessibilité aux études, de la recherche et du développement régional. Toutes les universités québécoises ont considéré les adultes comme un public-cible.

5. La commission se prononce en faveur de la gratuité scolaire à l'université, mais pour des raisons pratiques (pression sur les dépenses publiques), elle convient de retarder son application. Ces frais seront gelés pendant plus de 20 ans. 
Avant la réforme, les universités classiques avaient déjà un pied dans la formation des adultes. Pour McLean (2008 et 2010), dans les universités canadiennes, l'extension de l'enseignement a permis à des adultes l'accès aux études et le rattrapage scolaire. Cela a permis d'incarner l'idée de la deuxième chance, ainsi que la formation continue en lien à la demande des ordres professionnels et des employeurs publics et privés, en appui des études à temps partiel. Le Québec ne se démarque pas de cette situation, si ce n'est que ses universités ajoutent une originalité institutionnelle : le certificat, un diplôme universitaire de premier cycle équivalent à une année académique à temps plein et valant 30 crédits universitaires (60 ECTS).

Entre 1925 et 1960, toutes les universités québécoises ont créé des services dits d'extension de l'enseignement qui proposaient des cours permettant à des adultes d'obtenir le diplôme d'accès aux études universitaires. Dans les années 1960, les universités sont invitées à réaliser des activités de formation de niveau universitaire pour les étudiants et non des activités de préparation aux études universitaires ; ce rôle est désormais dévolu aux Cégeps ${ }^{6}$ nouvellement créés. Dès lors, les services d'extension ont progressivement disparu ou se sont convertis en services d'éducation des adultes, ciblant les adultes et élargissant leur offre. L'Université de Montréal (UDEM) créé ainsi une faculté d'éducation permanente. Alors que les autres facultés se centrent sur les programmes de cours en journée, cette faculté construit des programmes crédités qui peuvent se rapprocher de ceux offerts en journée, mais qui sont proposés le soir ou en fin de semaine.

Un doyen de l'éducation des adultes de l'Université de Montréal (2011) relate ainsi :

"Nous n'avons pas de corps professoral associé à notre faculté, on travaille avec le corps professoral des autres facultés selon une méthodologie orientée "marché", c'est-à-dire une analyse des besoins du marché du travail, des sociétés, et sur cette base on développe des filières de formation dans lesquelles on a pu identifier un intérêt. Ces pratiques qui existent depuis quarante ans nous permettent de développer des programmes ciblés sur des besoins. On va chercher des expertises en interne ou à l'extérieur et on travaille sur l'interdisciplinarité, à la marge des disciplines. "

Les constituantes de l'UQ ont mis en œuvre une institutionnalisation dite intégrée de l'éducation des adultes dans tous les programmes d'études, la responsabilité de l'éducation des adultes relevant des programmes et des départements.

Un chef de service de l'UQAM (2011) déclare ainsi :

" "Adulte".. On n'a pas choisi ce vocabulaire. On a même choisi de ne pas considérer les candidats adultes autrement que nos candidats réguliers. Quand ils font leur demande d'admission à l'université, ils n'ont pas à se qualifier d'adulte ou pas. Sauf qu'il y a des critères sur lesquels on les admet, ce que l'on appelle les "bases d'admission", les études collégiales évi-

6. Collèges d'enseignement général et professionnel. 
demment, la base des crédits universitaires et enfin la base de l'expérience (...) C'est sûr que c'est pour des gens aux profils moins traditionnels que l'on a créé la base expérience qui s'adresse aux plus de 21 ans. Ce que l'on voulait, cétait leur faire une place, mais ils ont le même choix de cours et de programmes et sur les mêmes créneaux horaires. Les gens sont mixés dans les classes. "

Au total, les universités ont toutes développé des dispositifs favorables à l'accessibilité aux études créditées (études à temps partiel, diplômes courts, admission sur l'expérience, calendrier des enseignements en trimestre...), dans une logique d'accessibilité de tous les publics, sans distinction.

Les programmes pensés pour les adultes sont des certificats. Le cumul de trois certificats permet l'obtention du baccalauréat (équivalent de la licence française). À l'entrée dans le XXIe siècle, en parallèle de l'élévation du niveau de formation de la population québécoise, les universités ont multiplié les programmes courts de second cycle (DESS - diplôme d'études supérieures spécialisées, programmes courts ou microprogrammes). Même à l'UDEM, où les facultés avaient pu laisser la responsabilité de l'accessibilité aux adultes à la Faculté d'éducation permanente, toutes les composantes se sont mises à développer une offre de second cycle en format court et enseigné le soir.

Un directeur de Bureau de recherche institutionnelle, UQAM (2011), déclare :

"Maintenant, les gens qui entrent sur le marché du travail, ils ont déjà un baccalauréat. Donc, si on veut les amener à une formation continue, ils nont pas envie de sinscrire en premier cycle. Alors, on crée des programmes de second cycle qui sont un peu plus valorisants pour eux, qui amènent des améliorations salariales, et ne soyons pas dupes, qui amènent des revenus pour les universités. Car on reçoit [du ministère] plus d'argent pour un étudiant de second cycle qu'un étudiant de premier cycle. C'est plus payant de créer un programme de deuxième cycle."

En outre, la création des certificats, et aujourd'hui celle des programmes courts de second cycle ont été l'occasion, pour les universités, d'innover, de diversifier et de professionnaliser l'ensemble de leurs programmes. Un autre mécanisme associé aux certificats a rapidement contribué à élargir le bassin de recrutement des étudiants adultes : les centres d'études universitaires et les sites hors campus qui ont dispensé des cours ailleurs que sur les campus principaux des universités (Corbo, 1994).

Ces délocalisations se sont accélérées depuis les années 2000 alors que les règles de financement des universités accentuaient l'importance du financement selon le nombre d'étudiants. La conséquence a été un accroissement de la concurrence interuniversitaire dans la quête aux étudiants. Ainsi, la grande région de Montréal accueille une majorité de ces centres d'études et pour plusieurs, le campus principal se situe dans d'autres régions du Québec.

Un permanent du Conseil supérieur de l'éducation, Québec (2011) déclare ainsi :

"Chez nous, c'est le terrain de la compétition : "envahissons le marché". Historiquement, on a eu les constituantes de l'Université du Québec dont on a dit, à tort, qu'elles avaient une vocation 
régionale. Ces universités sont aussi des pôles de développement national, voire international. L'université d'Ottawa a des panneaux pour recruter à la sortie du métro de l'UQAM et l'université de Sherbrooke est logée à l'autre bord du fleuve, à Longueuil [banlieue de Montréal]. "

En parallèle à cette offre de programmes et de modalités favorables à la présence des adultes dans le secteur des cours crédités, une autre innovation institutionnelle est apparue : les services à la collectivité. Des structures relativement petites au sein des universités ont pour mandat de réaliser des formations non-créditées et des activités de recherche en partenariat avec des groupes dont l'accès aux ressources universitaires est en théorie faible : centrales syndicales, groupes de femmes, groupes communautaires et groupes de citoyens.

\subsection{Une professionnalisation endogène et ses marges}

L'éducation des adultes a été un facteur de démocratisation de l'accès aux études postsecondaires. En effet, l'ouverture des universités à des étudiants plus âgés (figure 1), en situation de retour aux études, inscrits le plus souvent à temps partiel, s'est largement réalisée en accord avec des motivations liées à l'emploi et dans l'espoir d'une mobilité professionnelle. Certains programmes ont aussi été fréquentés par des groupes professionnels en processus «d'universitarisation" (Fourdrignier, 2009), comme les infirmières et les enseignants dans les années 1970.

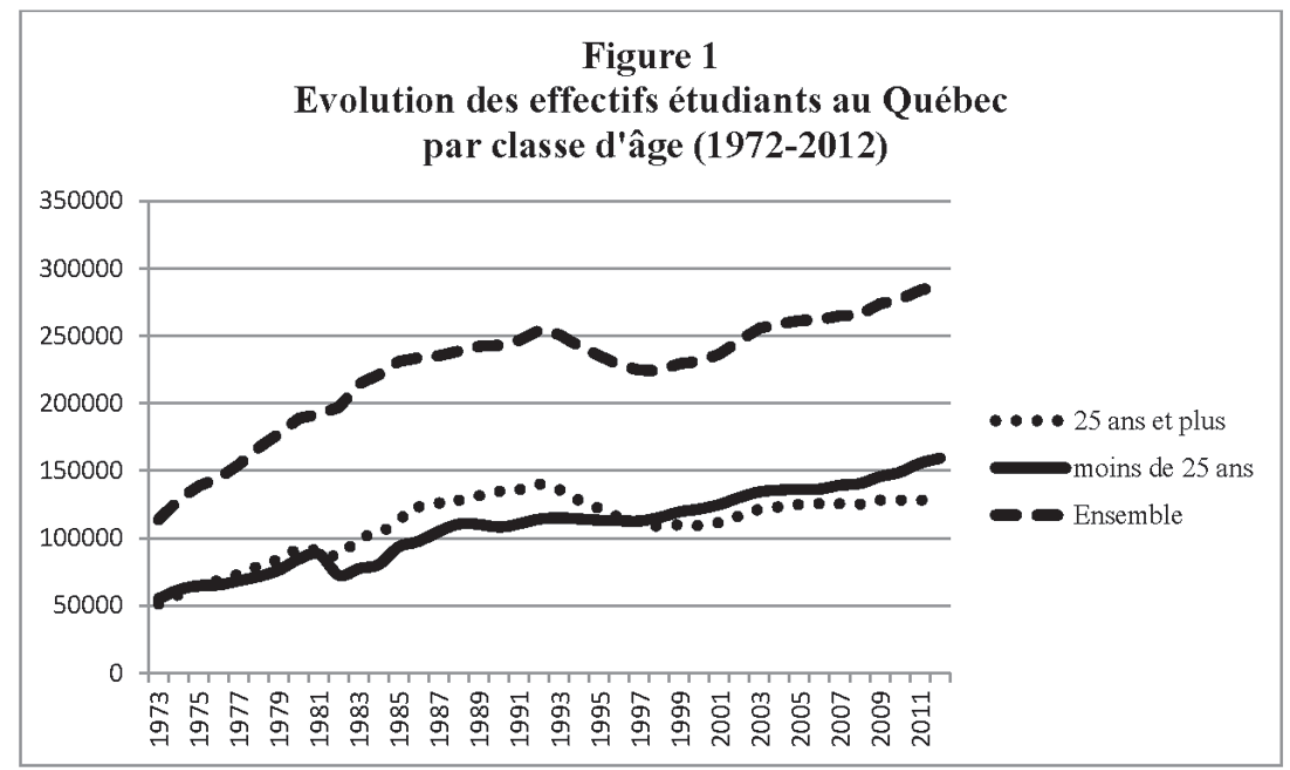

Source : données issues du système de Gestion des données sur les effectifs universitaires (GDEU) du gouvernement du Québec. 
Therrien (1997) rappelle que plusieurs programmes du soir, pensés pour les adultes, n'avaient pas de correspondance directe en formation de jour (ex. toxicomanie), pour des formations à des carrières en émergence (ex. les relations publiques), pour le perfectionnement de certaines catégories professionnelles en lien avec l'élévation des exigences scolaires de ces professions ou bien en raison de l'évolution rapide des technologies et des savoirs (administration, santé, éducation, etc.).

Sur la base de sondages auprès des étudiants de plus de 25 ans inscrits à l'Université de Montréal, en 1976, puis en 1979, il ressort que pour 71 \% d'entre eux, l'insertion scolaire est associée à leur activité de travail. Leur répartition par domaine d'études souligne l'attractivité des domaines professionnels. Les programmes en administration recueillent le plus de suffrages ( $43 \%$ en 1979), suivent les sciences humaines (19\%), les sciences de l'éducation (18\%) et la santé (9\%) (Granger, 1980 ; Therrien, Doray et Paquet, 1980). Des informations plus récentes tirées des données administratives (2000 et 2016) montrent que l'administration obtient toujours le plus de suffrages des plus de 25 ans (30\%), viennent ensuite les sciences humaines (15\%) et les sciences appliquées (12 à $13 \%$ ). Pour les moins de 25 ans, on observe une distribution plus étendue (aucun domaine n'obtient plus de $20 \%$ des suffrages) et un certain alignement avec les options prises par les plus âgés.

La professionnalisation a pris un autre visage à la fin de la décennie 1980 et au début des années 1990, avec la mise en ouvre d'une politique de soutien à la formation parrainée ou sur-mesure. Ces activités sont en grande partie non créditées, planifiées et organisées à la suite de demandes d'entreprises, d'ordres professionnels ou d'autres organisations et elles sont destinées à leurs salariés ou à leurs membres. Cette politique tient en partie à une volonté du gouvernement fédéral de modifier le financement de la formation de la main-d'œuvre en soutenant directement les employeurs plutôt que les institutions d'enseignement. L'objectif est d'orienter les ressources éducatives vers les besoins des entreprises en vue du retour à une croissance économique continue. Le gouvernement québécois s'opposant à ce changement, un compromis a été trouvé en développant dans les différents ordres d'enseignement, dont les universités, des services de formation sur-mesure à destination des entreprises, des administrations et des organisations professionnelles.

Cette politique a connu une institutionnalisation plus forte lors de l'adoption, en 1995, d'une loi favorisant le développement des compétences et la formation de la main-d'œuvre. Les entreprises dont la masse salariale atteint un certain seuil doivent dépenser (ou investir) au moins $1 \%$ de la valeur de leur masse salariale pour la formation de leurs employés. Ces deux décisions gouvernementales ont conduit à créer un marché de la formation continue. Les étudiants inscrits à ces formations ne font pas partie de l'effectif étudiant comptabilisé à des fins de financement public. Les instances qui organisent ces formations, relevant de facultés ou de vice-rectorats, sont tenues à 
l'autofinancement. Les pressions pour accumuler des surplus financiers sont fortes, surtout en période de coupes budgétaires.

Le Conseil supérieur de l'éducation (CSE, 2010) ainsi qu'une recherche récente (Doray et al., 2017) soulignent la variété des pratiques mises en œuvre dans ce cadre, ainsi que les défis d'articulation des demandes avec l'expertise interne des universités. Cette offre parrainée - dont on va voir qu'elle constitue l'idéal-type du système français est méconnue statistiquement. La comptabilisation des participants à ces activités de formation non-créditée est très difficile à réaliser en raison de l'inexistence de données centralisées à l'échelle provinciale et de leur dispersion à l'intérieur des universités entre différents services. En outre, les acteurs des universités rechignent à divulguer des informations sur ces activités en raison de la forte concurrence qui structure le marché. Dès les années 2000, ce volet de la formation des adultes est devenu une priorité pour les acteurs institutionnels des établissements.

En somme, la professionnalisation des universités québécoises par la formation des adultes a d'abord pris le visage d'une formation créditée associée à des préoccupations professionnelles. Son institutionnalisation, dans les années 1960, s'appuie sur une tradition d'ouverture des universités aux adultes, c'est-à-dire d'étudiants en retour aux études. L'offre et la demande de formation se rejoignent pour façonner des programmes à caractère professionnel dont la teneur a changé au cours des années. La fin des années 1980 marque le début de l'institutionnalisation d'un circuit éducatif parallèle, la formation sur-mesure pour les entreprises et les organisations professionnelles, mise en œuvre dans des instances spécifiques et en fonction de régulations fort différentes de celles qui prévalent dans la formation créditée. Le caractère endogène de la professionnalisation est alors atténué par le poids des organisations économiques dans la planification des activités de formation, fondée sur une régulation conjointe.

\section{En France, une professionnalisation exogène}

En France, il convient d'être à la fois en dedans et en dehors du secteur universitaire pour appréhender les matrices cognitives et matérielles de la formation des adultes à l'université, rassemblées sous le syntagme formation professionnelle continue (FPC) (2.1.). La FPC établit les conditions d'une professionnalisation de l'université à caractère exogène, car la formation des adultes relève d'abord du champ de l'emploi et du travail (2.2.). Finalement, le service de formation des adultes à l'université se construit sur la base de tensions exogène/endogène ainsi que marché/secteur public (2.3.). 


\subsection{L'ambition de la formation professionnelle continue (1950-1971)}

L'activité de formation des adultes se structure selon des modalités exogènes à l'université, en raison de trois événements historiques, survenus entre 1950 et 1975 . D’abord, l'engouement, pendant l'entre-deux guerres et après la Seconde Guerre mondiale, pour l'éducation des adultes et l'éducation permanente, ne s'est pas traduit par un appel aux universités (Manifet, 2015). Les mouvements d'éducation populaire comme ceux en faveur de la formation dans les milieux de travail suspectent le secteur public de ne pas pouvoir répondre aux besoins de formation des adultes.

Ensuite, dans les années 1950, les facultés et les écoles d'ingénieurs participent, à des degrés divers, au mouvement en faveur des adultes par des cours du soir, par une offre de diplômes d'établissements de durée courte (certificats) et par diverses autres initiatives (Laot, 1999 et 2009). Des dispositifs contribuent à sensibiliser l'ensemble du secteur aux besoins des adultes, comme la reconnaissance du titre d'ingénieur sur la base d'acquis professionnels ou l'Examen spécial d'entrée à l'université (ESEU) qui ouvre l'université aux non-bacheliers et deviendra, en 1996, le Diplôme d'accès aux études universitaires (DAEU), un équivalent au baccalauréat. À l'époque, il existe à l'université deux statuts, celui d'étudiant, celui d'auditeur libre. Mais, à l'intérieur du statut d'étudiant et sur présentations de pièces justificatives, des aménagements sont possibles pour les étudiants-salariés (mi-temps, téléenseignement, examen final plutôt que contrôle continu) ainsi que pour ceux qui, pour différentes raisons familiales, professionnelles ou de résidence à l'étranger, ne peuvent pas être assidus.

Émanent aussi des facultés, les Instituts de la promotion supérieure du travail qui poursuivent différents objectifs et s'intéressent à différents publics : rapprocher les chercheurs des acteurs économiques, faire de la recherche pédagogique, former les formateurs, former les jeunes peu diplômés et les travailleurs dans un but de rattrapage et de promotion individuelle, former les syndicalistes ou les cadres de l'éducation populaire dans un but de promotion collective ${ }^{7}$.

On observe néanmoins que, progressivement et à partir des années 1960, les activités des universités se concentrent sur la formation initiale et le développement de la recherche. Les politiques successives de démocratisation scolaire visent à élever le niveau d'études des jeunes générations ; ce principe se répercute de l'école à l'université. En matière de formation, les universités concentrent leurs efforts sur la gestion de la croissance continue du public en formation initiale. La situation est critique à la fin des années 1960. Elle le redeviendra entre la fin des années 1980 et le milieu des années 1990 (figure 2). Au milieu des années 1990, la France est un des pays de l'OCDE qui maintient le plus grand nombre de jeunes dans le système de formation initiale entre

7. Le CUCES (Centre universitaire de coopération économique et sociale), créé à Nancy en 1954, est le plus connu. 
20 et 24 ans et les jeunes français valorisent encore largement les études pour ellesmêmes (Galland, 2000). La diversification et la professionnalisation des contenus de formation sont des moyens de la scolarisation de masse avant de devenir des priorités au moins équivalentes à partir de la décennie 1990. Les dispositifs mis en place dans les années 1950, et favorables à l'éducation permanente, ne sont pas supprimés, mais ils sont peu valorisés et ne sont pas développés.

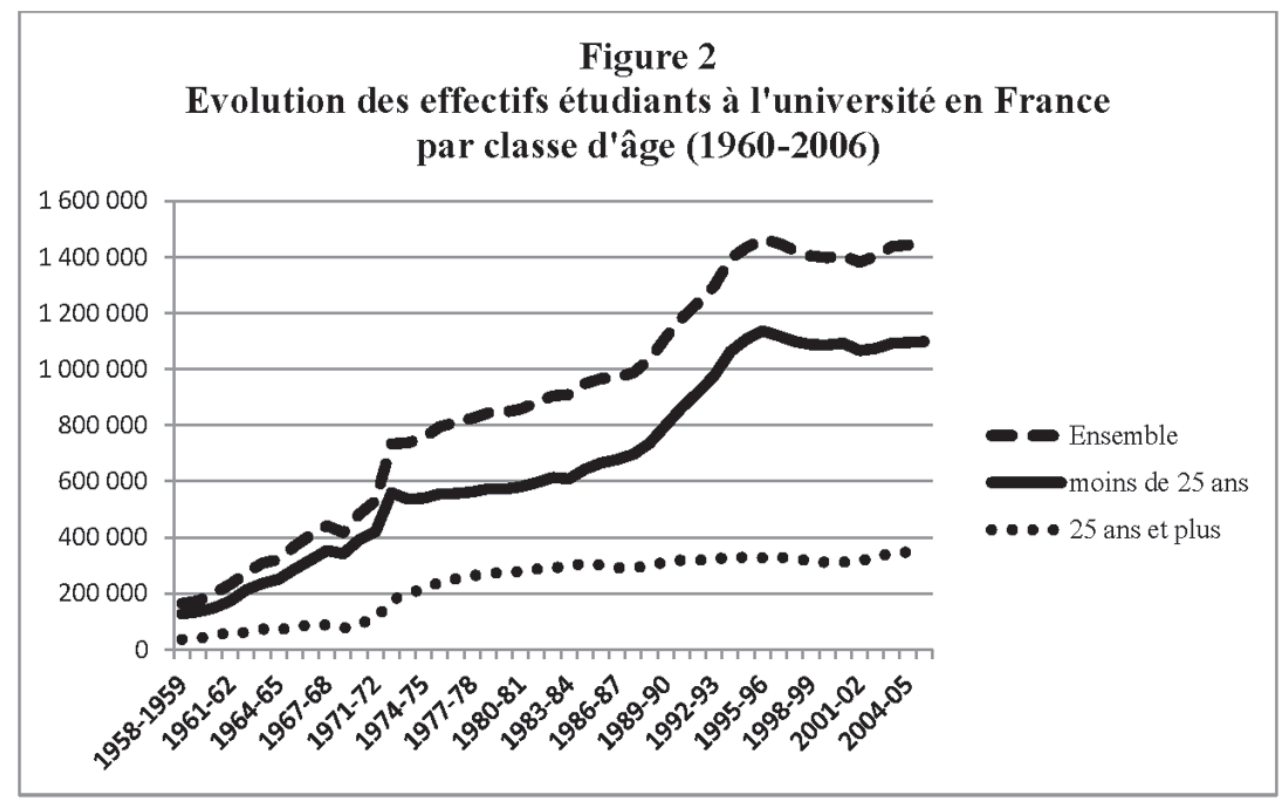

Source : données créées à partir du Système d'information et de suivi des Étudiants (SISE) du ministère de l'Enseignement supérieur français pour les années postérieures à 2005 et à partir d'autres publications ministérielles pour les années antérieures, « Informations statistiques » et « Tableaux statistiques » notamment.

Finalement, l'intégration de la formation pour adultes dans les politiques publiques accentue la mise à distance des universités de la gestion des besoins de formation des adultes. Alors que dans les années 1950, des lois instituent la promotion sociale des salariés, valorisent la formation dans un sens large et reconnaissent divers types de formation (Dubar et Gadéa, 1999), en 1971, la loi sur la formation professionnelle continue dans le cadre de l'éducation permanente, conçue comme un volet d'une politique globale, va devenir prépondérante et orienter les actions vers les milieux de travail. Basée sur un accord impliquant patronat et syndicats, cette loi marque un tournant en instituant un droit à la formation des salariés et un devoir de financement des employeurs, selon deux modalités : l'accès au congé-formation et le plan de formation de l'entreprise, le premier ayant connu des difficultés d'institutionnalisation, en particulier pour accéder à la formation universitaire. 
Cette politique vise à apaiser les relations au travail et la formation continue devient un instrument de gestion du rapport salarial. Qui plus est, elle exprime une volonté de changer l'État, sous couvert du slogan de "société nouvelle ", en passant d'une conception volontariste de son action à une conception partenariale, qualifiée de "socialedémocrate" (Dubar, 2004, p. 18). Sous cet angle, cette loi marque aussi un tournant dans le champ de l'éducation. Elle donne corps à une nouvelle politique éducative, externalisée par rapport au champ éducatif, car co-pilotée par le ministère du Travail et les partenaires sociaux que sont les syndicats d'employeurs et de salariés, puis les Conseils régionaux, depuis les années 1980. Elle institue un quasi-marché de la formation. Les fournisseurs de formation, privés et publics, dont les missions principales sont la formation ou non, sont tenus de respecter les règles édictées, sans distinction de statuts et de missions, et pour l'intérêt des besoins en compétences des employeurs et des travailleurs.

\subsection{Dans les universités, un service à part de formation continue}

La mise en œuvre de la FPC a généré l'espoir d’une professionnalisation généralisée de l'offre universitaire par l'implantation de règles de production, de sources d'inspiration et de revenus externes, issus de l'univers de l'emploi (Feutrie, 2002). Or, les effets de cette politique ont plutôt été de complexifier le service public universitaire par la segmentation des publics et la cohabitation de règles de droit différenciées. La formation continue et les adultes ont été marginalisés dans les universités.

Un premier décret (1984) porte sur la reconnaissance des acquis de l'expérience professionnelle pour accéder à tous les niveaux de l'enseignement supérieur. Les décennies suivantes, la politique de validation des acquis s'est étoffée et élargie vers une logique de validation de niveaux de diplômes. Un second décret (1985) légifère sur des modes spécifiques de financement et de gestion de la formation continue universitaire, tels que l'obligation d'autofinancement et l'établissement d'une comptabilité séparée. Dans ce nouveau cadre, l'appellation adulte n'est pas d'usage. Les personnes qui ont interrompu leurs études et qui sont actifs, en emploi ou en recherche d'emploi et qui s'inscrivent à l'université prennent en effet le statut de stagiaire. Leur formation est payante et les tarifs doivent s'aligner sur les coûts réels. Les stagiaires signent une convention avec l'université et financent leurs études grâce aux cotisations des employeurs et/ou sur leurs fonds propres.

L'adjonction de ce monde, nouveau et à part, de la formation continue au sein des établissements, ouvre de nouvelles discussions, comme par exemple à Toulouse, sur les aménagements des études et des examens pour les étudiants salariés ou placés dans d'autres situations les empêchant d'être assidus. Or, ces réflexions ne sont pas intégrées ou même simplement reliées au nouvel enjeu posé par la loi de juillet 1971 sur la formation professionnelle continue, dont la mise en œuvre s'avère par ailleurs hésitante. 
Extrait de l'exposé du président, PV du conseil de l'université du 12 décembre 1972, université de Lettres et Sciences humaines de Toulouse :

"Le président a tenu à insister sur la mission fondamentale de l'université en matière de formation continue, même si elle n'en a pas le monopole. Si l'université refusait de participer à cet effort indispensable de remise à jour des connaissances que la nation attend d'elle, elle se priverait d'une occasion unique de renouvellement. La crainte d'une dévaluation des formations universitaires ne paraît pas fondée, surtout si l'on considère que les universités ne sont pas obligées de faire de la formation continue à n'importe quel niveau puisqu'elles n'en ont pas le monopole. De plus, il faut éviter que les universitaires soient contraints d'aller ailleurs pour faire de la formation continue, car l'université se doit d'assurer directement sa double fonction dans ce domaine, à la fois culturelle et de recyclage proprement dit (amélioration de la qualification)."

Des services communs de formation continue sont le plus souvent créés dans les établissements qui prennent en charge cette mission, en appui des entités pédagogiques dans le meilleur des cas, ou en parallèle et en réponse au faible investissement des personnels enseignants le plus souvent. Leur rôle est délicat. Ils ne sont en effet pas censés produire de façon autonome une offre de formation, mais "valoriser " les ressources disponibles dans les universités tout en ayant une approche marché (Manifet, 2012) - par exemple, leurs formateurs doivent se répartir de manière équitable entre enseignants de l'université et intervenants externes reconnus pour leurs compétences dans le domaine professionnel considéré.

Un directeur de service commun, université pluridisciplinaire, France (2007) déclare ainsi :

"Je crois que la ligne directrice de notre service (...) on essaie de faire que toute notre organisation soit bâtie de telle façon qu'elle réponde à la demande qui émerge. Le problème, c'est de cerner la demande et de ne pas partir de notre offre. Ceci étant dit, il arrive que des pans entiers de notre offre soient en rapport avec cette demande, mais que cette offre ne soit pas assez connue. C'est donc aussi cette offre qu'il faut savoir promouvoir, pour la faire connaître."

Dans tous les cas, le développement de cette activité de formation continue, selon les termes de la loi de 1971, dépend largement des dispositifs et des moyens alloués tirés du champ du travail et de l'emploi. Pour obtenir le statut de stagiaire à l'université, le candidat, salarié, indépendant, chef d'entreprise ou demandeur d'emploi doit d'abord répondre aux conditions statutaires d'éligibilité établies par la politique de FPC et ses principaux acteurs (les employeurs pour le plan de formation, les commissions paritaires et les Organismes paritaires collecteurs agrées - OPCA - pour le congé individuel de formation - CIF -, les Régions et Pôle Emploi pour les demandeurs d'emploi) ${ }^{8}$. Le

8. Les CIF constituent la formule la plus adaptée pour l'université : ils ouvrent la possibilité de formation sur 
développement de la formation continue universitaire dépend ensuite des dispositifs émanant du champ universitaire (procédures de validation ou de dispense de titre, offre adaptée et aménagée, information et communication, conseil et orientation).

\subsection{Une professionnalisation exogène sous tension}

La professionnalisation de l'université par la politique de formation continue prend finalement deux formes polarisées, marchandisation de l'offre à destination des adultes et/ou scolarisation d'une partie du public adulte. La formation continue inscrit d'abord les universités sur le marché de la FPC, répondant aux demandes émanant des milieux de travail et des acteurs publics. Elle contribue ainsi, par son caractère marginal ${ }^{9}$ et/ ou " marchandisé " (Agulhon, 2004 ; Caillaud, 2007) aux " glissements " de toute la politique de FPC (Dubar, 2004) par rapport à ses objectifs initiaux : la prépondérance des logiques économiques et la minorisation des logiques de promotion sociale ; une politique des prescripteurs publics (service public de l'emploi et régions) sous contrainte d'une situation de crise, focalisée sur l'emploi, la lutte contre le chômage, le placement ou le maintien dans l'emploi ; la marchandisation des opérateurs placés en concurrence et construisant leur offre en fonction des critères et des fonds de la FPC.

Bien que la formation continue soit parmi les trois missions universitaires fondamentales et que les évaluations successives enjoignent les établissements à vraiment investir cette mission, les universités n'ont jamais été considérées ni positionnées comme des acteurs privilégiés de la FPC. Le dernier rapport de l'Inspection générale de l'administration de l'Education nationale et de la Recherche (IGAENR) le souligne, tout en promouvant " une plus grande prise en compte des universités dans la gouvernance régionale» (2014, pp. 88-93). Le dialogue entre les partenaires sociaux se fait sans elles et leur est défavorable. Les publics-cibles de la politique de FPC sont ceux pour lesquels l'offre universitaire est moins attractive ou désignée inadéquate (salariés du secteur privé plutôt que du secteur public, formations courtes et ciblées, jeunes et adultes aux faibles qualifications). Enfin, les conditions de mise en œuvre de l'offre de formation tendent à assimiler les fournisseurs de formation à des opérateurs de formation au service de

une offre plus large, sur un temps plus long et sur le temps de travail. Mais il n'y a pas l'assurance que ce droit s'accompagne d'un maintien de la rémunération. C'est l'OPCA auquel cotise l'entreprise au titre du CIF qui décide. Jusqu'en 2014, le droit individuel à la formation octroyait $20 \mathrm{~h}$ de formation/an avec possibilité de cumul sur six ans et plafond à 120 heures, le plus souvent hors du temps de travail, le soir ou le week-end, en lien étroit avec l'emploi occupé et financées par l'entreprise. Depuis 2015, il a été remplacé par le compte personnel de formation qui peut rouvrir les possibilités de formation à l'université.

9. Les établissements d'enseignement supérieur dans leur ensemble (universités, CNAM - Conservatoire national des arts et métier -, écoles) représentent près de $3 \%$ du chiffre d'affaires des opérateurs de FPC (Grille, 2014). Ce pourcentage représente 448000 stagiaires qui se répartissent entre des programmes préparant aux diplômes nationaux et ouverts à la formation continue (30\% des stagiaires), des diplômes d'universités adaptés à la formation continue $(22 \%)$, des stages courts $(23 \%)$ et des conférences à caractère culturel (21\%). 
l'adaptabilité et de l'employabilité des individus. La tendance actuelle n'est pas à une mise en question du compromis fonctionnel, mais plutôt à son élargissement, puisque désormais les règles de la FPC s'étendent au public de la formation initiale (formation par alternance ou par apprentissage).

L'engagement variable des universités et l'attrait que peut receler le marché de la FPC pour leur financement conduit à des disparités fortes entre établissements. Aujourd'hui, dix-neuf universités sur près de 80 accueillent $50 \%$ des stagiaires de la formation continue universitaire et réalisent $52 \%$ du chiffre d'affaire du secteur (IGAENR 2014, p. 25). Ces "conquérants" (Manifet, 2012) développent différentes stratégies de développement de leur offre de formation continue : une offre courte non diplômante à destination des salariés en plan de formation et financée par les entreprises ; une offre plutôt institutionnelle et sociale, financée par les prescripteurs publics et à destination des publics salariés aidés ou en recherche d'emploi ; une offre fortement orientée vers la demande individuelle, ciblée sur l'offre diplômante et à caractère culturel, financée plutôt par les particuliers.

Cependant, la marchandisation évacue un pan de la demande individuelle des adultes à laquelle les universités répondent par des pratiques inclusives non prévues ou écartées par la politique de FPC. Le ministère de l'Enseignement supérieur lui-même a créé, dans les années 2000, un nouveau statut d'inscription à l'université : celui de reprise d'études sans financement, pour tenter de rendre visible cette demande adulte dissimulée sous le statut " étudiant " et admettre à demi-mot une prise en charge sur son budget. Du côté des établissements, ces pratiques inclusives peuvent exprimer une revendication de savoir et de pouvoir répondre de façon endogène aux besoins de formation des adultes et des employeurs. C'est ainsi que la majorité des universités françaises vantent aux candidats stagiaires le fait que toute leur offre de formation initiale est ouverte à la formation continue. Elles valorisent les formations d'un an et plus ainsi que les formations diplômantes, dont les calendriers et les horaires exigent une disponibilité pendant les jours et les heures normalement travaillées dans l'entreprise.

\section{Quelles sont les dimensions de la professionnalisation 3 de l'université ?}

Le travail de reconstitution comparé des ancrages intellectuels, institutionnels et organisationnels de la formation des adultes à l'université, en France et au Québec, permet de comprendre des trajectoires singulières de professionnalisation, tout en révélant des catégories transversales aux deux sociétés : les singularités offrent la possibilité de rapprochements analytiques et la montée en généralité (Figure 3).

Au Québec, l'idéal de l'éducation permanente a été repris par les acteurs éducatifs de tous les ordres d'enseignement. Le mode de professionnalisation est endogène parce que 
les réformes inscrivent le développement de l'éducation permanente dans les institutions scolaires, dont les universités. La professionnalisation y est présente par la gamme de programmes destinés à un public adulte, dont la participation est fortement orientée par l'emploi. Les adultes sont intégrés aux programmes crédités, souvent pensés pour eux. La formation des adultes a joué un rôle dans la diversification et la professionnalisation des programmes universitaires dans leur ensemble.

En France, au contraire, la professionnalisation des formations universitaires (Gayraud, Simon-Zarca et Soldano, 2011) n'est pas dépendante des stratégies en faveur des adultes, bien qu'il faille tenir compte du fait que, désormais, le modèle de la FPC régule aussi le processus de professionnalisation de la formation initiale. Ce modèle a, à l'origine, construit une professionnalisation à part, ciblée sur les adultes, et qualifiée d'exogène. Il s'agit de produire une offre de formation conçue de façon conventionnelle entre employeurs, prescripteurs, stagiaires et universités. De ce point de vue, la configuration française fonctionne sur une division polaire, comme l'a déjà montré Éric Verdier en qualifiant le régime français d' "académique " : " tout se passe avant 25 ans puis l'entreprise adapte les compétences des individus à ses besoins avec le soutien actif des pouvoirs publics" (2008, p. 216). Cette dualité formation initiale/formation continue n'a pas facilité les négociations de normes de la formation pour adultes et des programmes universitaires traditionnels. Elle a en outre alimenté la focalisation des instances régulières des universités sur leur public traditionnel, jeune et en continuité de formation.

Avant tout, la formation des adultes se pose comme un enjeu sociétal de part et d'autre de l'Atlantique et de ce point de vue, il y a convergence. Les objectifs associés sont similaires ainsi que variés, révélant un système complexe d'intérêts. L'importance des études est reconnue pour l'intérêt personnel des adultes, l'avancement de carrière et la mobilité sociale, mais aussi pour la croissance des entreprises, le développement social et économique et la cohésion des nations. Le débat sur l'éducation des adultes fût relativement concomitant au Canada et en France, au cours des années 1950-60, en lien avec la prise en charge de ces questions par l'UNESCO. On note alors que, dans les deux cas, l'articulation formation-emploi ne constitue pas à elle seule un enjeu assez englobant pour mettre l'ensemble de la société en mouvement. C'est l'idéal de l'éducation permanente qui tient ce rôle, intégrant l'articulation formation-emploi dans un schème plus large associant l'éducation et les autres univers sociaux, économiques et politiques, ainsi que les enjeux de promotion individuelle, de développement économique et de cohésion sociétale. 
Figure 3 : Trajectoires et catégories de la professionnalisation des universités : la formation pour adultes

\begin{tabular}{|c|c|c|c|}
\hline $\begin{array}{l}\text { Échelles de structuration de la } \\
\text { professionnalisation }\end{array}$ & $\begin{array}{l}\text { Les catégories de la } \\
\text { professionnalisation }\end{array}$ & $\begin{array}{c}\text { Québec } \\
\text { Professionnalisation } \\
\text { endogène }\end{array}$ & $\begin{array}{c}\text { France } \\
\text { Professionnalisation } \\
\text { exogène }\end{array}$ \\
\hline Échelle sociétale & $\begin{array}{c}\text { La projection sur laquelle se construit } \\
\text { le consensus sociétal }\end{array}$ & $\begin{array}{c}\text { Éducation permanente puis } \\
\text { Formation tout au long de la vie }\end{array}$ & \begin{tabular}{|c} 
Éducation permanente puis \\
Formation tout au long de la vie
\end{tabular} \\
\hline $\begin{array}{l}\text { Échelle étatique (ou système } \\
\text { politique) }\end{array}$ & $\begin{array}{c}\text { La gouvernance : la place, le rôle } \\
\text { de l'État } \\
\end{array}$ & « Révolution tranquille » & «Société nouvelle » \\
\hline Échelle intersectorielle & L'articulation Formation \& Emploi & $\begin{array}{c}\text { Convention libérale } \\
\text { «Éducation des adultes » }\end{array}$ & $\begin{array}{c}\text { Convention Fonctionnelle } \\
\text { "Formation professionnelle } \\
\text { continue» } \\
\end{array}$ \\
\hline Échelle sectorielle & $\begin{array}{l}\text { La fonction, la place et le rôle des } \\
\text { universités }\end{array}$ & Institution ouverte & Institution sanctuaire \\
\hline \multirow[t]{2}{*}{$\begin{array}{l}\text { Échelle des universités } \\
\text { (Institution, Organisation, } \\
\text { Établissement) }\end{array}$} & $\begin{array}{c}\text { Construction institutionnelle des } \\
\text { besoins et des attentes de l'adulte : } \\
\text { quelle conception de l'individu et de } \\
\text { sa place dans le système de formation } \\
\text { et de production? }\end{array}$ & $\begin{array}{l}\text { Finalité de l'adulte = profession } \\
\text { perspective, individuelle (service } \\
\text { à la clientèle) et intégrative (non } \\
\text { segmentation des publics) }\end{array}$ & $\begin{array}{c}\text { Finalité de l'adulte = l'emploi } \\
\text { perspective structurelle (réponse } \\
\text { aux employeurs et prescripteurs) } \\
\text { et différenciée (création d'un } \\
\text { segment « stagiaire ») }\end{array}$ \\
\hline & $\begin{array}{l}\text { Dispositifs d'aménagement des } \\
\text { contenus et des parcours d'études: } \\
\text { quelle conception des savoirs à } \\
\text { transmettre? }\end{array}$ & \begin{tabular}{|c|} 
Plutôt parcours aménagé \\
Temps partiel, certificats, calendrier \\
trimestriel, cours du soir et de fin de \\
semaine, valorisation de l'expérience \\
pour l'accès aux études \\
\end{tabular} & $\begin{array}{l}\text { Plutôt contenu aménagé } \\
\text { Service commun de formation } \\
\text { continue comme sas d'entrée } \\
\text { et premier accueil, stages } \\
\text { courts, diplômes d'université, } \\
\text { masters professionnels ouverts } \\
\text { à la FC, validation des acquis de } \\
\text { l'expérience }\end{array}$ \\
\hline
\end{tabular}

Source : Tableau synthétique créé par les auteurs.

En continuité, l'enjeu de l'éducation permanente se traduit, dans les deux sociétés, par une réflexion sur le rôle et la place de l'État et s'oriente vers une voie alternative de gouvernance étatique associée à des projets de société ambitieux - révolution tranquille au Québec, société nouvelle en France - qualifiés tous deux de sociaux-démocrates. Il se trouve que ces idées similaires s'inscrivent dans des trajectoires sociétales. Au Québec, il s'agit de renforcer l'État dans son rôle d'encadrement de la société et du système éducatif et en France, de décentraliser sa place dans la société et le système éducatif.

Ensuite, les liens entre éducation, emploi et travail sont problématiques à construire dans tous les cas, mais donnent lieu à des arbitrages qui different. La hiérarchie des valeurs associées à l'éducation permanente est par exemple divergente et valorise finalement deux conceptions de la professionnalisation. La gestion du rapport politique entre éducation, travail et emploi est plutôt « libérale " au Québec (Schuller et al., 2002), fondée sur une convention selon laquelle l'école est garante d'une protection face à la demande d'emploi 
tout en étant un outil de mobilité sociale, alors qu'elle est plutôt " fonctionnelle » en France parce qu'on accorde une priorité à la préparation à l'emploi ${ }^{10}$.

Enfin, une autre convention d'ordre sociétal et étatique, imbriquée aux précédentes, concerne la fonction, la place et le rôle des universités dans la société, ainsi que leur rapport à l'État. D’un côté, le gouvernement et la société font confiance aux universités pour répondre à cet enjeu, de l'autre moins. C'est sans doute que, d'un côté, l'Université bénéficie d'une image d'institution ouverte. Les universités québécoises, à l'instar des universités nord-américaines, fonctionnent sur un principe d'autonomie vis-à-vis du politique. Elles ont investi l'éducation des adultes de longue date, non pas à la suite d'une injonction politique, mais parce qu'il s'agit d'un espace d'opportunité de développement, convergent avec l'enjeu politique de la démocratisation des études universitaires. En France, au contraire, du fait d'une intégration plus étroite à l'appareil d'État, on reproche aux acteurs du secteur de fonctionner sur une logique d'institution-sanctuaire. De plus, les priorités de la politique nationale de démocratisation scolaire conduisent les universités à concentrer leurs efforts sur les publics jeunes dans un objectif d'accessibilité. Dans ce contexte, l'autre demande politique de s'engager dans la formation professionnelle continue, selon une articulation fonctionnelle apparaît secondaire et impose un grand écart par rapport aux principes et aux normes dominantes des activités universitaires. L'institution Université résonne donc différemment dans les deux sociétés en fonction des modalités du rapport société/université et du rôle d'intermédiation assuré par le système politique.

À l'échelle des universités, les besoins et les attentes du public adulte comme les savoirs à transmettre ne sont pas rationalisés de la même manière. Ainsi, le développement d'une culture de la demande adulte a pris deux orientations distinctes : celle d'une culture du public adulte (Québec), celle d'une culture de la demande du monde du travail (France). Dans un cas, on se préoccupe particulièrement de l'aménagement des parcours, dans l'autre, de celui des contenus. Ces deux orientations, l'une orientée vers les personnes, l'autre plutôt organisationnelle, ne sont pas sans lien avec des définitions distinctes de ce qu'est le travail (Dubar et Tripier, 2005). La perspective québécoise s'approche plutôt du sens anglo-saxon de la profession, c'est-à-dire un ensemble de personnes exerçant un même métier pour lequel la professionnalisation consiste en la construction d'une qualification par un diplôme universitaire. La vision française assimilerait la profession à l'emploi que l'on occupe et à la fonction que l'on exerce dans une organisation (Fourdrignier, 2009).

\section{Conclusion}

L'approche sociétale de la comparaison internationale a permis d'appréhender comment un enjeu commun, la professionnalisation des universités, se décline en des compositions complexes de valeurs, d'intérêts, d'acteurs et de dispositifs à différentes échelles de

10. Une autre possibilité serait l'autonomisme radical (Radical separatists) qui ne fait aucun lien entre éducation, emploi et travail. 
régulation à partir desquelles il a été possible de dégager des cohérences sociétales. La comparaison intervient alors comme une étape nécessaire à la théorisation, permettant l'émergence de catégories générales concernant la professionnalisation des universités et à partir desquelles les singularités prennent tout leur sens.

Les configurations sociétales sont loin d'être figées et leurs singularités ne sont ni exemplaires, ni l'expression d'une essence culturelle, ni déterminées par les moments fondateurs (Révolution tranquille ou Société nouvelle). Mais elles se construisent historiquement à partir de décisions collectives et de processus de rationalisation, d'institutionnalisation et de normalisation des pratiques (Powell et Maggio 1997).

À cet égard, les deux sociétés observées offrent la possibilité, après les rapprochements analytiques, d'opérer des rapprochements empiriques. Après les accords fondateurs des années 1960 et 1970, est-on arrivé à un tournant dans les modalités du rapprochement formation-emploi à l'Université et dans les deux sociétés étudiées ? L'environnement politique, économique et social des universités fait pression sur celles-ci pour qu'elles changent, qu'elles se repositionnent et assument des fonctions nouvelles en matière de formation tout au long de la vie (Nesbit, Dunlop \& Gibson, 2007).

On observe une certaine convergence en faveur de conventions de type fonctionnel. Au Québec, ce type de convention est devenu une norme équivalente à la convention libérale, avec la formation sur-mesure. Cette orientation s'explique en grande partie par les changements des politiques publiques des pays occidentaux qui ont visé le retour à une croissance économique continue tout en s'adossant à des figures "d'Etat-marché » (Muller 2004), prégnantes en matière éducative.

Ces orientations en faveur de l'offre parrainée sont désormais communes aux deux sociétés, pour des raisons également financières et pour faire face à l'austérité budgétaire dans les secteurs publics. Il se trouve que les catégories nationales de la formation continue universitaire sont perméables aux transferts issus d'autres situations nationales ou de modèles internationaux. Or, l'UNESCO n'est plus le seul référent international des pratiques nationales en matière de formation pour adultes, au profit de l'entrée en scène de l'OCDE et de la Commission européenne, dont les systèmes d'information ont récemment intégré des indicateurs ayant trait à la formation des adultes (Eurostudent Eurostat, 2009 ; OCDE, 2013). En parallèle, le concept de formation tout au long de la vie remplace celui de l'éducation permanente et se décline socialement par une attention plus grande (Québec) ou accentuée (France) accordée à la pertinence des formations par rapport au marché de l'emploi.

Il n'est pas dit qu'une telle orientation soit tenable et efficace. La professionnalisation des universités est une question éminemment politique (Verdier, 2008, p. 203) qui suppose des arbitrages sur des questions majeures, d'abord sur les projets de société à partir desquels peut se justifier la conduite d'un rapprochement entre formation et emploi. Quand bien même ce rapprochement est quasi-naturalisé dans les sociétés contemporaines mar- 
quées par le poids symbolique et matériel de l'économie (en référence à Karl Polanyi) (Chambard et Cozanet, 2016), il fait toujours l'objet de débats, de confrontations et/ou de négociations et les compromis institués ne sont ni stéréotypés, ni unilatéraux, mais relèvent d'équilibres tenables entre différents espaces sociaux, activités, intérêts, valeurs et normes. La professionnalisation des universités (Lessard et Bourdoncle, 2002) engage des arbitrages sur les responsabilités partagées de l'État, du système économique et du système d'enseignement, sur la place et le rôle d'intermédiation des systèmes politiques, sur la fonction, la place et le rôle des universités dans la société et leur dépendance/autonomie à l'égard des systèmes politique et économique, sur la fonction, la place et le rôle du travail et de l'emploi, et enfin sur la place accordée aux individus dans les systèmes de formation et de production.

\section{Bibliographie}

Agulhon C. (2004), "La formation continue à l'Université ", Cahiers de la recherche sur l'éducation et les savoirs, $\mathrm{n}^{\circ} 3$, pp. 193-214.

Beaupère N., Bosse N. \& Lemistre P. (2014), « Expérimenter pour généraliser le Portefeuille d'Expériences et de Compétences à l'université : le sens de l'évaluation ", Formation Emploi, n 126, pp. 99-117.

Caillaud P. (2007), « La construction d'un droit de la formation professionnelle des adultes » in Brucy et al., Former pour réformer. Retour sur la formation permanente (1945-2004), Paris, La Découverte, pp. 171-210.

Chambard O. \& Cozanet L. (2016), «Introduction : Nouveaux éclairages sur les relations entre enseignement supérieur et monde économique ", Formation Emploi, n 132 , pp. $7-13$.

Corbo C. (1994), Matériaux fragmentaires pour une histoire de l'UQAM, Montréal, Éditions Logiques.

Crespo M. (2013), L'université se professionnalise-t-elle au Canada? Une analyse longitudinale des choix de programmes de formation, Montréal, CIRANO.

CSE (Conseil supérieur de l'éducation) (2010), Les services offerts aux entreprises par le réseau de l'éducation : pour un meilleur accès aux ressources collectives, Avis de la commission de l'éducation des adultes et de la formation continue, Québec, Gouvernement du Québec.

Demazière O. (2002), "Chômeurs "âgés" et chômeurs "trop vieux", articulation des catégories gestionnaires et interprétatives ", Sociétés comtemporaines, vol. 4, n 48, pp. 109-130.

Doray P., Simoneau F. B. \& Solar-Pelletier L. (2017), L'adéquation entre la formation et l'emploi : le cas de la formation sur mesure dans l'enseignement supérieur à Montréal, Rapport 
remis à la CPMT dans le cadre du Programme de recherche sociale appliquée, Montréal, UQAM.

Doray P., Tremblay E. \& Groleau A. (2015), «Quelle professionnalisation dans les universités québécoises ", Formation Emploi, n 129, pp. 47-64.

Dubar C. et Tripier P. (2005), Sociologie des professions, Paris, Armand Colin (2eme édition augmentée).

Dubar C. (2004), La formation professionnelle continue, Paris, La Découverte, "Coll. Repères " (1ere éd. 1984).

Dubar C. \& Gadéa P. (1999), La promotion sociale en France, Paris, Presses universitaires Septentrion.

Dubar C., Gadea C. \& Rolle C. (2003), « Pour une analyse comparée des configurations : réflexions sur le cas de la formation continue » in Lallement M. et Spurdk, J., Stratégies de la comparaison internationale, Paris, Éditions du CNRS, pp. 57-70

Dubois V. (2003), « La sociologie de l'action publique : De la socio-histoire à l'observation des pratiques (et vice-versa) » in Laborier P. et Trom D. (Dir.), Historicités de l'action publique, Paris, Presses universitaires de France, pp. 347-364.

Dubois V. (2012), La politique culturelle: genèse d'une catégorie d'intervention publique, Paris, Belin.

Eurostudent Eurostat (2009), The Bologna process in Higher education in Europe, Eurostat, Commission européenne.

Ferretti L. (1994), L'université en réseau. Les 25 ans de l'université du Québec, Presses de l'Université du Québec.

Feutrie M. (2002), « La formation continue : d'une activité à la marge à la mobilisation de toute l'université " in Pennec S., Former des adultes: l'Université et les transformations de l'emploi, Rennes, Presses universitaires de Rennes, pp. 123-139.

Fourdrignier M. (2009) « Professionnaliser les métiers du sanitaire et du social à l'université : une mission impossible ? ", Formation Emploi, n 108, décembre, pp. 67-81.

Galland O. (2000), "L'allongement de la jeunesse en Europe », Revue de l'OFCE, n 72 , janvier, pp. 187-191.

Gally N. (2012), «Écrire le contraste au-delà des typologies : l'apport de l'histoire croisée à la comparaison internationale ", Revue internationale de politique comparée, vol. 19, $\mathrm{n}^{\circ} 1$, pp. 19-38.

Gayraud L., Simon-Zarca G. \& Soldano C. (2011), « Université : les défis de la professionnalisation ", NefCéreq : http://www.cereq.fr. 
Granger D. (1980), Profils professionnels et académique des étudiants inscrits à des programmes pour adultes et à des cours libres du premier cycle ainsi que de ceux inscrits à des cours réguliers du premier cycle à l'université de Montréal et à ses écoles affiliées à l'automne 1979, Montréal, Centre de sondage de l'Université de Montréal.

Grille J. (2014), « La formation continue dans l'enseignement supérieur », L'état de l'Enseignement supérieur et de la Recherche en France, n 7, avril, pp. 46-47.

IGAENR (2014), Limplication des universités dans la formation tout au long de la vie, Paris, ministère de l'Éducation.

Kolsaker A. (2013), "Relocating Professionalism in an English University", Journal of Higher Education Policy and management, vol. 36, n 2, pp. 129-142.

Landrier S. \& Nakhili N. (2012), "Comment l'orientation contribue aux inégalités de parcours scolaires en France ", Formation Emploi, n 109, pp. 23-36.

Laot F. (1999), La formation des adultes: histoire d'une utopie en acte - le complexe de Nancy, Paris, L'Harmattan.

Laot F. (2009), «Un modèle universitaire et régional de formation d'adultes dans les années 1960 : l'épisode oublié des Aurefa et leur échec face à la loi de 1971 ", Éducation et Sociétés, vol. 2, n 24, pp. 143-157.

Lemistre P. (2016). "La professionnalisation des formations initiales : une solution aux mutations du marché de l'emploi pour les jeunes ? ", Revue française de pédagogie, vol. 3, $n^{\circ} 192$, pp. 61-72.

Lessard C. \& Bourdoncle R. (2002), " Note de synthèse : Qu'est-ce qu'une formation professionnelle universitaire ? Conceptions de l'université et formation professionnelle ", Revue française de pédagogie, vol. 139, 1, pp. 131- 53.

Manifet C. (20I2), «La formation continue universitaire en France : un lieu de redéfinition du service public éducatif ", Éducation et Sociétés, vol. 30, nº 2, pp. 183-200.

Manifet C. (2015), "L'éducation des adultes dans les universités en France. Une mission hétérodoxe ?", Cahiers de la recherche sur l'éducation et les savoirs, n 14, pp. 267-294.

Maurice M. (1989), "Méthode comparative et analyse sociétale. Les implications théoriques des comparaisons internationales ", Sociologie du travail, vol. 31, n 2, pp. 175-193.

Maurice M., F. Sellier \& J.-J. Silvestre (1982), Politique d'éducation et organisation industrielle en France et en Allemagne : essai d'analyse sociétale, Paris, Presses universitaires de France.

McLean S. (2008), "Extending resources, fostering progress, or meeting needs? University extension and continuing education in western Canada", British Journal of Sociology of Education, vol. 29, n 1, pp. 91-103. 
McLean S. (2010), «Essor, chute, puis renaissance des études à temps partiel pour adultes à l'Université de Montréal ", Revue canadienne de L'éducation permanente universitaire, vol. 37, $\mathrm{n}^{\circ} 1$, pp. 1-16.

Muller P. (2004), « L'État en action revisité », Pôle Sud, vol. 2, n² 1, pp. 31-42.

Nesbit T., Dunlop C. \& Gibson L. (2007), "Lifelong Learning in Institutions of Higher Education", Canadian Journal of University Continuing Education, vol. 33, n 1, pp. 35-60.

OCDE (2013), Perspectives de l'OCDE sur les compétences 2013 : Premiers résultats de l'évaluation des compétences des adultes, Éditions OCDE.

Parent A. M. (1964), Rapport de la Commission royale d'enquête sur l'enseignement dans la province de Québec, Québec, Gouvernement du Québec.

Powell W.W. \& Di Maggio P.J. (1997), «Le néo-institutionnalisme dans l’analyse des organisations ", Politix, vol. 10, n 40, pp. 113-54.

Ryan C. (1964), Rapportdu Comité d'étudesurl'éducation des adultes, Québec, Gouvernement du Québec.

Schuller T., Istance D. \& Schuete H. G. (2002), International Perspectives on Lifelong Learning, From Recurrent Education to the Knowledge Society, London, The Society for Research into Higher Education and Open University Press.

Therrien R. (1997), "Éléments de réflexion sur le champ de l'éducation des adultes à l'université " in Chenard P., Lévolution de la population étudiante à l'université. Facteurs explicatifs et enjeux, Québec, Presses de l'université du Québec, pp. 105-118.

Therrien R., Doray P. \& Paquet P. (1980), Les caractéristiques démographiques des étudiants des programmes pour adultes, Montréal, document de recherche, Faculté de l'Éducation Permanente, Université de Montréal.

Tremblay A. (1962), Rapport du Comité d'étude sur l'enseignement technique et professionnel, Québec, Gouvernement du Québec.

Verdier É. (2008), "L'éducation et la formation tout au long de la vie : une orientation européenne, des régimes d'action publique et des modèles nationaux en évolution ", Sociologie et Sociétés, vol. 40, n 1, pp. 195-225.

Von Rosenbladt B. (2010), "Adult education and training in comparative perspective _ Indicators of participation and country profiles ", Statistics in transition-New edition, vol. 11, n³, pp. 465-502.

Wittorsky R. (2012), "La professionnalisation de l'offre de formation universitaire : quelques spécificités ", Revue internationale de pédagogie de l'enseignement supérieur [en ligne], vol. $28, \mathrm{n}^{\circ} 1,13 \mathrm{p}$. 


\section{Négociations Conflit, décision et délibération}

\section{No27 - Été 2017}

Équité et expertise dans les négociations environnementales

Michel BOURBAN, Justice climatique et négociations internationales - Alain GRANDJEAN, "Imaginer que les savants ou experts puissent prendre de "bonnes" décisions pour la collectivité est une naïeté dangereuse » (Entretien) - Laurent FABIUS, "Il n'y a pas d'accord si tout le monde n'est pas d'accord » (Entretien)

Varia

Emmanuel VIVET, « Dans un monde où le multiatéralisme fonctionne mal, lUnion européenne reste un exemple positif de delibération entre États et d'effectivité des décisions prises » (Entretien)

Nouvelles approches de la décision collective : compromis, consensus, vote

Stéphanie NOVAK et Philippe URFALINO, Nowelles approcbes de la décision collective : une introduction - Sandrine BAUME, What place sbould compromises be given in democracy? A reflection on Hans Kelsen's contributions - Stéphanie NOVAK, La prise de décision peut-elle être informelle ? - Eva KRICK, The Myth of Effective Veto Power under the Rule of Consensus. Dymamics and Democratic Legitimacy of Collective Decision-Making by "Tacit Consent" - Tanja PRITZLAFF-SCHEELE, Between "apparent unanimity" and majority vote. A political micro-ethnograpby of committee decision-making - Philippe URFALINO, La nature du vote oral. Leçons d'une étude de cas

Bonnes feuilles

Jeremy BENTHAM, Essai de tactique politique

Négociations est disponible en texte intégral sur www.cairn.info

Négociations. Une revue en langue française, dont l'ambition est d'instruire la question de la négociation dans la diversité de ses dimensions, en favorisant la confrontation interdisciplinaire et en faisant se rejo indre plusieurs traditions d'étude.

Site de la revue : www.cairn.info/revue-negociations.htm

Directeurs de la publication : Aurélien COLSON, ESSEC-Irené et Christian THUDER0Z, INSA de Lyon, Centre Max Weber 